\title{
$\beta$-Cyclodextrin catalyzed synthesis of substituted indoles in aqueous medium
}

\author{
Jilla Shankar, Gaddam Satish, Bandam Santosh Pavan Anil Kumar \\ and Yadavalli Venkata Durga Nageswar* \\ Council of Scientific Industrial Research-Medicinal Chemistry and Pharmacology Division, Indian Institute of Chemical Technology, Uppal Road, Hyderabad 500-007, \\ India \\ *Corresponding author at: Council of Scientific Industrial Research-Medicinal Chemistry and Pharmacology Division, Indian Institute of Chemical Technology, Uppal \\ Road, Hyderabad 500-007, India. \\ Tel.: +91.040.27191654. Fax: +91.040.27193189. E-mail address: dryvdnageswar@gmail.com (Y.V.D. Nageswar).
}

\section{COMMUNICATION INFORMATION}

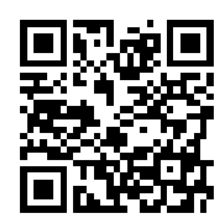

DOI: $10.5155 /$ eurjchem.5.4.668-670.1085

Received: 28 April 2014

Received in revised form: 16 June 2014

Accepted: 16 June 2014

Online: 31 December 2014

\section{KEYWORDS}

\section{Indole}

$\beta$-Cyclodextrin

$N$-Methylaniline

Aqueous medium

$\mathrm{N}$-Methylbenzamines

Aldehyde derivatives

\section{Introduction}

Multicomponent reactions (MCRs) are of increasing importance in organic and medicinal chemistry and have offered many fascinating and challenging transformations in organic synthesis [1-7]. These multicomponent reactions are emerging as powerful tools for the synthesis of biologically important compounds [8-9]. 3-Substituted indole moieties are important as they are widely distributed in nature and reveal a broad range of biological activities [10]. Indoles with amino alkyl/aryl substituents at the 3-position are considered as potential pharmacophores [11] in drug discovery and are found in various natural products [12], useful in the treatment of migraine, breast cancer [13], and HIV-1 [14]. The immense potential of indole nucleus for development of drug candidates, prompted many synthetic chemists to explore different methodologies suitable for the synthesis of 3-substituted indoles.

In view of different biological activities associated with indole derivatives and in continuation of our interest in the use of cyclodextrins as mild and efficient biomimetic catalysts in promoting various organic transformations [15-22], we here in report the synthesis of indole derivatives by the reaction of indole with different aldehydes and $N$-methylaniline under neutral conditions involving $\beta$-cyclodextrin in water medium.

\section{Experimental}

\subsection{Instrumentation}

All the products were characterized by their NMR and Mass spectra. ${ }^{1} \mathrm{H}$ NMR is recorded on Varian 200 spectrometer $(200$ $\mathrm{MHz}$, in $\left.\mathrm{CDCl}_{3}\right)$ and the chemical shifts were reported in parts per million (ppm, $\delta$ ) downfield from tetramethylsilane. IR was recorded on a Thermo Nicolet Nexus 670 FT-IR spectrometer Mass spectra were obtained using a VG Autospec mass spectrometer.

\subsection{General procedure for the synthesis of $N$ - $((1 H-i n d o l-3-y l)$ (phenyl) methyl)-N-methylbenzenamine}

All chemicals were purchased from Fluka and S. D. Fine Chemicals and directly used for the synthesis. Analytical Thin Layer Chromatography (TLC) was carried out by using silica gel $60 \mathrm{~F}_{254}$ pre-coated plates. Visualization was accomplished with UV lamp or I2 stain. All reactions were carried out without any special precautions in an atmosphere of air. 


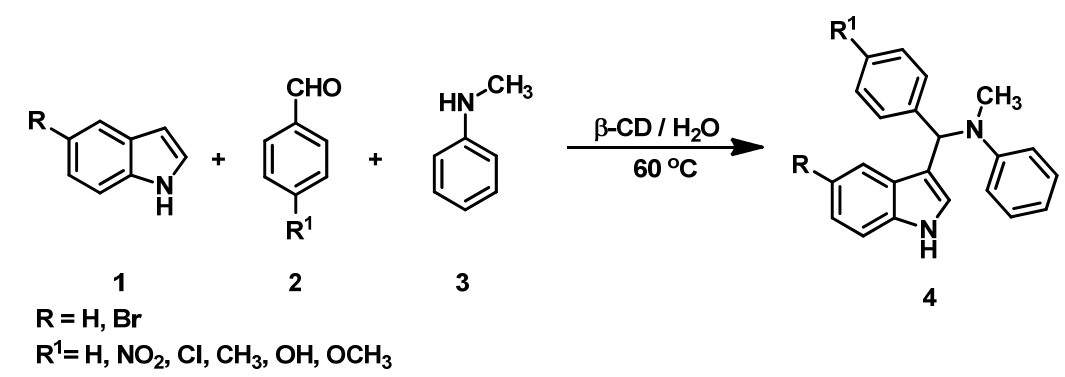

Scheme 1

\begin{tabular}{|c|c|c|c|c|c|}
\hline Entry & $\mathbf{R}$ & $\mathbf{R}^{1}$ & Product & Time (h) & Yield (\%) b \\
\hline 1 & $\mathrm{H}$ & $\mathrm{H}$ & $4 a$ & 4.0 & 86 \\
\hline 2 & $\mathrm{H}$ & $4-\mathrm{Cl}$ & $4 \mathrm{~b}$ & $\begin{array}{l}4.0 \\
3.5\end{array}$ & 84 \\
\hline 3 & $\mathrm{H}$ & $4-\mathrm{CH}_{3}$ & $4 \mathrm{c}$ & 4.5 & 86 \\
\hline 4 & $\mathrm{H}$ & $4-\mathrm{NO}_{2}$ & $4 d$ & 4.5 & 85 \\
\hline 5 & $\mathrm{H}$ & $4-\mathrm{OCH}_{3}$ & $4 \mathrm{e}$ & 4.5 & 83 \\
\hline 6 & $\mathrm{H}$ & $4-\mathrm{OH}$ & $4 \mathrm{f}$ & 4.5 & 81 \\
\hline 7 & $\mathrm{Br}$ & $4-\mathrm{NO}_{2}$ & $4 \mathrm{~g}$ & 4.5 & 86 \\
\hline 8 & $\mathrm{Br}$ & $4-\mathrm{OCH}_{3}$ & $4 \mathrm{~h}$ & 4.5 & 85 \\
\hline 9 & $\mathrm{Br}$ & $4-\mathrm{Cl}$ & $4 \mathrm{i}$ & 3.5 & 80 \\
\hline 10 & $\mathrm{Br}$ & $4-\mathrm{CH}_{3}$ & $4 \mathrm{j}$ & 4.5 & 85 \\
\hline
\end{tabular}

a Reaction conditions: Aldehyde $(1.0 \mathrm{mmol}), N$-methylaniline $(1.0 \mathrm{mmol})$, indole $(1.0 \mathrm{mmol}), \beta$-cyclodextrin $(10 \mathrm{~mol} \%), 60{ }^{\circ} \mathrm{C}$.

b Isolated yield.

$\beta$-Cyclodextrin (1.135 g, $1 \mathrm{mmol}$ ) was dissolved in water $(15 \mathrm{~mL})$ by warming up to $60{ }^{\circ} \mathrm{C}$ until a clear solution was formed. To this clear solution, aldehyde $(1.0 \mathrm{mmol})$ was added and stirred for $10 \mathrm{~min}$, and then $N$-methylaniline $(1.0 \mathrm{mmol})$ was added. After stirring for half an hour 1 equivalent of indole (1.0 mmol) was added. The reaction mixture was stirred until completion of the reaction as indicated by TLC. The reaction mixture was cooled and $\beta-\mathrm{CD}$ was filtered. The aqueous phase was extracted with ethyl acetate $(3 \times 10 \mathrm{~mL})$ and the organic layers were washed with water, saturated brine solution, and dried over anhydrous $\mathrm{Na}_{2} \mathrm{SO}_{4}$. The combined organic layers were evaporated under reduced pressure and the resulting crude product was purified by column chromatography by using ethyl acetate: hexane $(2: 8, v: v)$ as eluent to give the corresponding $\mathrm{N}$-((1H-indol-3-yl) (phenyl) methyl)- $N$-methyl benzeneamine as pure product in good yield (Scheme 1).

$N$-((1H-Indol-3-yl) (phenyl) methyl)- $N$-methylbenzenamine (Table 1, entry 1): Yield: $86 \%$. ${ }^{1} \mathrm{H}$ NMR $\left(200 \mathrm{MHz}, \mathrm{CDCl}_{3}, \delta\right.$, ppm): 8.00 (s, 1H, NH, Pyr), 7.84 (s, 1H, CH, Pyr.), 7.15-7.36 (m, 8H, Ar-H), 7.0-7.12 (m, 3H, Ar-H), 6.47-6.60 (d, $J=9.06 \mathrm{~Hz}, 3 \mathrm{H}$, Ar-H), 5.57 (s, 1H, CH), $2.82\left(\mathrm{~s}, 3 \mathrm{H}, \mathrm{CH}_{3}\right)$. MS (ESI, $\left.m / z\right): 313$ $[\mathrm{M}+\mathrm{H}]^{+}$.

$N$-((4-Chlorophenyl) (1H-indol-3-yl) methyl)- $N$-methyl benzeneamine (Table 1, entry 2): Yield: $84 \% .{ }^{1} \mathrm{H}$ NMR $(200$ $\mathrm{MHz}_{\mathrm{CDCl}}, \delta, \mathrm{ppm}$ ): 8.01(s, 1H, NH, Pyr), 7.96 (s, 1H, CH, Pyr.), 7.04-7.26 (m, 7H, Ar-H), 6.91-7.02 (d, $J=8.49 \mathrm{~Hz}, 2 \mathrm{H}, \mathrm{Ar}-\mathrm{H})$, 6.39-6.53 (m, 4H, Ar-H), $5.48(\mathrm{~s}, 1 \mathrm{H}, \mathrm{CH}), 2.72\left(\mathrm{~s}, 3 \mathrm{H}, \mathrm{CH}_{3}\right)$. MS (ESI, $m / z): 347[\mathrm{M}+\mathrm{H}]^{+}$.

$N$-((1H-Indol-3-yl) (p-tolyl) methyl)- $N$-methylbenzenamine (Table 1, entry 3): Yield: $86 \% .{ }^{1} \mathrm{H}$ NMR $\left(200 \mathrm{MHz}, \mathrm{CDCl}_{3}, \delta\right.$, ppm): 8.00 (s, 1H, NH, Pyr), 7.90 (s, 1H, CH, Pyr.), 7.36-7.39 (d, $2 \mathrm{H}, J=8.49 \mathrm{~Hz}, \mathrm{Ar}-\mathrm{H}), 7.19-7.23(\mathrm{~m}, 3 \mathrm{H}, \mathrm{Ar}-\mathrm{H}), 6.93-7.16$ (m, 5H, Ar-H), 6.49-6.52 (d, J = 7.52 Hz, 3H, Ar-H), 5.51 (s, 1H, CH), $2.77\left(\mathrm{~s}, 3 \mathrm{H}, \mathrm{CH}_{3}\right), 2.29\left(\mathrm{~s}, 3 \mathrm{H}, \mathrm{CH}_{3}\right)$. MS (ESI, $\left.\mathrm{m} / \mathrm{z}\right): 327[\mathrm{M}+\mathrm{H}]+$.

$N$-((1H-Indol-3-yl) (4-nitrophenyl) methyl)- $N$-methyl benzeneamine (Table 1, entry 4): Yield: $85 \% .{ }^{1} \mathrm{H}$ NMR $(200$ $\left.\mathrm{MHz}, \mathrm{CDCl}_{3}, \delta, \mathrm{ppm}\right): 8.30$ (s, 1H, NH, Pyr), 8.25 (s, 1H, CH, Pyr.), 7.23-7.35 (m, 5H, Ar-H) 7.09-7.15 (m, 2H, Ar-H), 6.916.99 (d, J=8.1 Hz, 3H, Ar-H), 6.44-6.55 (d, J=6.79 Hz, 3H, Ar-H), $5.58(\mathrm{~s}, 1 \mathrm{H}, \mathrm{CH}), 2.78\left(\mathrm{~s}, 3 \mathrm{H}, \mathrm{CH}_{3}\right)$. MS (ESI, $\left.m / z\right): 380[\mathrm{M}+\mathrm{Na}]^{+}$.

$N$-((1H-Indol-3-yl) (4-methoxyphenyl) methyl)- $N$-methyl benzenamine (Table 1, entry 5): Yield: 83\%. ${ }^{1} \mathrm{H}$ NMR (200 MHz,
$\mathrm{CDCl}_{3}, \delta, \mathrm{ppm}$ ): 8.01 (s, 1H, NH, Pyr), 7.89 (s, 1H, CH, Pyr.), 7.47$7.60(\mathrm{~m}, 4 \mathrm{H}, \mathrm{Ar}-\mathrm{H}), 6.94-7.18(\mathrm{~m}, 5 \mathrm{H}, \mathrm{Ar}-\mathrm{H}), 6.47-6.52(\mathrm{~d}, J=$ $8.30 \mathrm{~Hz}, 4 \mathrm{H}, \mathrm{Ar}-\mathrm{H}), 5.54(\mathrm{~s}, 1 \mathrm{H}, \mathrm{CH}), 3.80\left(\mathrm{~s}, 3 \mathrm{H}, \mathrm{OCH}_{3}\right), 2.78(\mathrm{~s}$, $\left.3 \mathrm{H}, \mathrm{CH}_{3}\right)$. MS (ESI, $\left.m / z\right): 343[\mathrm{M}+\mathrm{H}]+$.

4-((1H-Indol-3-yl) (methyl (phenyl) amino) methyl) phenol (Table 1, entry 6): Yield: $81 \% .{ }^{1} \mathrm{H}$ NMR $\left(200 \mathrm{MHz}, \mathrm{CDCl}_{3}, \delta\right.$, ppm): 8.01 (s, 1H, NH, Pyr), 7.71 (s, 1H, CH, Pyr.), 7.31-7.36 (d, J $=7.55 \mathrm{~Hz}, 2 \mathrm{H}, \mathrm{Ar}-\mathrm{H}), 7.20-7.26(\mathrm{~d}, J=8.30 \mathrm{~Hz}, 3 \mathrm{H}, \mathrm{Ar}-\mathrm{H}), 6.91-$ $7.15(\mathrm{~m}, 6 \mathrm{H}, \mathrm{Ar}-\mathrm{H}), 6.60-6.65(\mathrm{~d}, J=9.01 \mathrm{~Hz}, 2 \mathrm{H}, \mathrm{Ar}-\mathrm{H}), 5.47(\mathrm{~s}$, $1 \mathrm{H}, \mathrm{CH}), 5.37(\mathrm{~s}, 1 \mathrm{H}, \mathrm{OH}), 2.16\left(\mathrm{~s}, 3 \mathrm{H}, \mathrm{CH}_{3}\right)$. MS (ESI, $\left.m / z\right): 329$ $[\mathrm{M}+\mathrm{H}]^{+}$.

$N$-((5-Bromo-1H-indol-3-yl) (4-nitrophenyl) methyl)- $N$ methylbenzenamine (Table 1, entry 7): Yield: $86 \% .{ }^{1} \mathrm{H}$ NMR $\left(200 \mathrm{MHz}, \mathrm{CDCl}_{3}, \delta, \mathrm{ppm}\right): 8.92$ (s, $\left.1 \mathrm{H}, \mathrm{NH}, \mathrm{Pyr}\right), 8.84$ (s, $1 \mathrm{H}, \mathrm{CH}$, Pyr.), 8.09-8.23 (m, 6H, Ar-H), 7.30-7.35 (t, $J=8.30 \mathrm{~Hz}, 3 \mathrm{H}, \mathrm{Ar}-$ H), 6.93(s, $1 \mathrm{H}, \mathrm{Ar}-\mathrm{H}), 6.49-6.59(\mathrm{~d}, J=8.32 \mathrm{~Hz}, 2 \mathrm{H}, \mathrm{Ar}-\mathrm{H}), 5.56(\mathrm{~s}$, $1 \mathrm{H}, \mathrm{CH}), 2.83\left(\mathrm{~s}, 3 \mathrm{H}, \mathrm{CH}_{3}\right)$. MS (ESI, $\left.m / z\right): 459[\mathrm{M}+\mathrm{Na}]^{+}$.

$\mathrm{N}$-((5-Bromo-1H-indol-3-yl) (4-methoxyphenyl)methyl)- $N$ methylbenzenamine (Table1, entry 8): Yield: $85 \% .{ }^{1} \mathrm{H}$ NMR $\left(200 \mathrm{MHz}, \mathrm{CDCl}_{3}, \delta, \mathrm{ppm}\right): 8.00$ (s, $\left.1 \mathrm{H}, \mathrm{NH}, \mathrm{Pyr}\right), 7.30$ (s, $1 \mathrm{H}, \mathrm{CH}$, Pyrro ), 7.10-7.71 (m, 6H, Ar-H), 6.98-7.05 (d, J=9.16 Hz, 2H, ArH), 6.71-6.75 (d, $J=8.25 \mathrm{~Hz}, 2 \mathrm{H}, \mathrm{Ar}-\mathrm{H}), 6.44-6.49(\mathrm{~d}, J=8.24 \mathrm{~Hz}$, $2 \mathrm{H}, \mathrm{Ar}-\mathrm{H}), 5.40(\mathrm{~s}, 1 \mathrm{H}, \mathrm{CH}), 3.71\left(\mathrm{~s}, 3 \mathrm{H}, \mathrm{OCH}_{3}\right), 2.76\left(\mathrm{~s}, 3 \mathrm{H}, \mathrm{CH}_{3}\right)$. MS (ESI, $m / z): 422[\mathrm{M}+\mathrm{H}]^{+}$.

$N$-((5-bromo- $1 H$-indol-3-yl) (4-chlorophenyl)methyl)- $N$ methylbenzenamine (Table 1, entry 9): Yield: $80 \% .{ }^{1} \mathrm{H}$ NMR $\left(200 \mathrm{MHz}, \mathrm{CDCl}_{3}, \delta, \mathrm{ppm}\right): 8.10(\mathrm{~s}, 1 \mathrm{H}, \mathrm{NH}, \mathrm{Pyr}), 8.00$ (s, $1 \mathrm{H}, \mathrm{CH}$, Pyr.), 7.05-7.32 (m, 6H, Ar-H), 6.92-6.94 (d, J=8.30 Hz, 2H, ArH), 6.47-6.53 (d, J = 8.30 Hz, 4H, Ar-H), $5.42(\mathrm{~s}, 1 \mathrm{H}, \mathrm{CH}), 2.75(\mathrm{~s}$, $3 \mathrm{H}, \mathrm{CH}_{3}$ ). MS (ESI, $\left.m / z\right): 426[\mathrm{M}+\mathrm{H}]^{+}$.

$N$-((5-bromo- $1 H$-indol-3-yl) (p-tolyl) methyl)- $N$-methyl aniline (Table 1, entry 10): Yield: 85\%. ${ }^{1} \mathrm{H}$ NMR $(200 \mathrm{MHz}$, $\mathrm{CDCl}_{3}, \delta, \mathrm{ppm}$ ): 8.00 (s, 1H, NH, Pyr), 7.95 (s, 1H, CH, Pyr.), 7.36 (s, 1H, Ar-H), 7.17-7.25 (m, 2H, Ar-H), 7.05 (s, 3H, Ar-H), 6.95$6.99(\mathrm{~d}, J=8.30 \mathrm{~Hz}, 3 \mathrm{H}, \mathrm{Ar}-\mathrm{H}), 6.49-6.53(\mathrm{~d}, J=8.30 \mathrm{~Hz}, 3 \mathrm{H}, \mathrm{Ar}-$ $\mathrm{H}), 5.44(\mathrm{~s}, 1 \mathrm{H}, \mathrm{CH}), 2.81\left(\mathrm{~s}, 3 \mathrm{H}, \mathrm{CH}_{3}\right), 2.31\left(\mathrm{~s}, 3 \mathrm{H}, \mathrm{CH}_{3}\right)$. MS (ESI, $m / z): 406[\mathrm{M}+\mathrm{H}]^{+}$.

\section{Results and discussion}

Cyclodextrins are cyclic oligosaccharides with hydrophobic cavities, and can bind substrates selectively and catalyze 
chemical reactions by supramolecular catalysis involving reversible host-guest complexation. We describe herein, the aqueous phase synthesis of indole derivatives demonstrating the remarkable catalytic activity of $\beta$-cyclodextrin (Scheme 1). In general, the reaction was carried out by the in situ formation of the $\beta$-CD complex of aldehyde in water followed by the addition of 1 equivalent of $N$-methylaniline and 1 equivalent of indole followed by subsequent stirring at $60{ }^{\circ} \mathrm{C}$ to give the corresponding $\quad N$-((1H-indol-3-yl)(phenyl)methyl)- $N$-methyl benzenamine in high yields.

The reaction goes to the completion in a short time (3.5-4.5 h). Several examples illustrating this simple and practical methodology are summarized in Table 1 . No byproduct formation was observed. $\beta$-Cyclodextrin can be easily recovered and reused. All the compounds were characterized by ${ }^{1} \mathrm{H}$ NMR, and mass spectrometry. The catalytic activity of cyclodextrin in these reactions was established by the fact that no reaction was observed in the absence of cyclodextrin. Evidence for the complexation between aldehyde and cyclodextrin is supported by ${ }^{1} \mathrm{H}$ NMR spectroscopy. The complexation with $\beta$-CD increases the reactivity of aldehyde functional group due to the intermolecular hydrogen bonding with the CD-hydroxyl groups facilitating the addition of $N$-methylaniline. Here, $\beta$-CD not only forms the inclusion complex with aldehyde but is also involved in the intermolecular hydrogen bonding with the guest to promote the reaction.

\section{Conclusion}

A neutral aqueous phase synthesis of substituted indoles was developed by the reaction of the corresponding aldehyde with $N$-methylaniline and indole promoted by $\beta$-cyclodextrin. These cyclodextrin-mediated aqueous phase reactions are very useful both from economical and environmental perspective. This straightforward and environmentally benign methodology may find wide spread application in organic and medicinal chemistry.

\section{Acknowledgement}

We thank Council of Scientific and Industrial Research, New Delhi, India, for fellowship to JillaShankar, Gaddam Satish and Bandam Santosh Pavan Anil Kumar.

\section{References}

[1]. Domling, A. Chem. Rev. 2006, 106, 17-89.

[2]. Tejedor, D.; Cruz, D. G.; Exposito, A. S.; Tellado, J. J. M.; Armas, P. D.; Tellado, F. G. Chem. Eur. J. 2005, 11, 3502-3510.

[3]. Ramon, D. J.; Yus, M. Angew. Chem. Int. Ed. 2005, 44, 1602-1634.

[4]. Simon, C.; Constantieux, T.; Rodriguez, J. Org. Chem. 2004, 24, 49574980.

[5]. Orru, R. V. A.; Michiel, D. G. Synthesis 2003, 1471-1499.

[6]. Bienayme, H.; Hulme, C.; Oddon, G.; Schmitt, P. Chem. Eur. J. 2000, 6, 3321-3329.

[7]. Ulaczyk-Lesanko, A.; Hall, D. G. Current Chem. Biol. 2005, 9, 266-276.

[8]. Weber, L. Curr. Med. Chem. 2002, 9, 2085-2093.

[9]. Hulme, H.; Gore, V. Curr. Med. Chem. 2003, 10, 51-80.

[10]. Jiang, B.; Yang, C. G.; Wang, J. J. Org. Chem. 2001, 66, 4865-4869.

[11]. Leze, M. P.; Le Borgne, M.; Marchand, P.; Loquet, D.; Kogler, M.; Le Baut, G.; Palusczak, A.; Hartmann, R. W. J. Med. Chem. 2004, 19, 549557.

[12]. Deng, J.; Sanchez, T.; Neamati, N.; Briggs, J. M. J. Med. Chem. 2006, 49, 1684-1692.

[13]. Wynne, J. H.; Stalick, W. M. J. Org. Chem. 2002, 67, 5850-5853.

[14]. Sundberg, R. J. Indoles, Academic Press, San Diego, 1996.

[15]. Shankar, J.; Karnakar, K.; Srinivas, B.; Nageswar, Y. V. D. Tetrahedron Lett. 2010, 51, 3938-3939.

[16]. Ramesh, K.; Murthy, S. N.; Karnakar. K.; Nageswar, Y. V. D. Tetrahedron Lett. 2011, 52, 4734-4737.

[17]. Ramesh, K.; Murthy, S. N.; Nageswar, Y. V. D. Tetrahedron Lett. 2011, 52, 2362-2366.

[18]. Ramesh, K.; Murthy, S. N.; Karnakar. K.; Nageswar, Y. V. D. Tetrahedron Lett. 2011, 52, 3937-3941.

[19]. Ramesh, K.; Karnakar, K.; Satish, G.; Reddy, K. H. V.; Nageswar, Y. V. D. Tetrahedron Lett. 2012, 53, 6095-6099.
[20]. Ramesh, K.; Karnakar, K.; Satish, G.; Anil Kumar, B. S. P.; Nageswar, Y. V. D. Tetrahedron Lett. 2012, 53, 6936-6939.

[21]. Ramesh, K.; Narayana Murthy, S.; Karnakar, K.; Nageswar, Y. V. D. Eur. J. Chem. 2012, 3(1), 119-124.

[22]. Swapna, K.; Narayana Murthy, S.; Nageswar, Y. V. D. Eur. J. Chem. 2011. 2(2), 272-275 